\title{
Liens utiles
}

\section{Pour effectuer des démarches ou télécharger des formulaires ${ }^{1}$}

\begin{tabular}{|c|c|}
\hline Démarche & URL \\
\hline Déclaration ou demande d'autorisation & http://www.asn.fr/index.php/Haut-de-page/Professionnels/ \\
\hline d'une installation & Formulaires $\# 2$ \\
\hline Gestion des NRD & https://basenrd.irsn.fr/ \\
\hline \multicolumn{2}{|c|}{$\begin{array}{l}\text { Déclaration d'un événement significatif http://www.vigie-radiotherapie.fr/ } \\
\text { de radiothérapie }\end{array}$} \\
\hline $\begin{array}{l}\text { Déclaration d'un événement significatif } \\
\text { de radioprotection (radiologie ou } \\
\text { médecine nucléaire) }\end{array}$ & $\begin{array}{l}\text { http://www.asn.fr/index.php/Haut-de-page/Professionnels/Les- } \\
\text { guides-de-declaration-des-evenements-significatifs/Guide-n-11-de- } \\
\text { declaration-des-evenements-significatifs-en-radioprotection-hors- } \\
\text { INB-et-TMR }\end{array}$ \\
\hline Déclaration de matériovigilance & $\begin{array}{l}\text { http://www.afssaps.fr/Activites/Comment-signaler-ou-declarer/ } \\
\text { Incident-ou-risque-d-incident-grave-lie-a-l-utilisation-un-dispositif- } \\
\text { medical-Materiovigilance/ } \% 28 \text { offset } \% 29 / 3 \\
\text { https://www.formulaires.modernisation.gouv.fr/gf/cerfa_10246.do }\end{array}$ \\
\hline $\begin{array}{l}\text { Demande de dérogation pour un } \\
\text { dispositif médical non marqué CE }\end{array}$ & $\begin{array}{l}\text { http://www.afssaps.fr/Activites/Mise-sur-le-marche-des-dispositifs- } \\
\text { medicaux-et-dispositifs-medicaux-de-diagnostic-in-vitro-DM- } \\
\text { DMIA-DMDIV/DM-et-DMIA-Procedures-specifiques/ }\end{array}$ \\
\hline
\end{tabular}

1. L'IRSN met à disposition une page donnant de nombreux liens utiles pour les activités professionnelles de physique médicale: http://www.irsn.fr/FR/professionnels_sante/liens_utiles/Pages/ default.aspx?Cible=ProfessionnelDeSante 


\section{Pour consulter ou télécharger des publications}

L'IRSN met à disposition une page permettant de télécharger de nombreux documents utiles pour les activités professionnelles de physique médicale : http://www.irsn.fr/FR/professionnels_sante/documentation/Pages/rapports.aspx Les liens donnés dans le tableau ci-dessous sont des adresses URL qui pointent directement sur les pages des sites où sont recensées des publications d'intérêt.

\begin{tabular}{|c|c|c|}
\hline Sigle & Nom & URL (publications) \\
\hline AAPM & $\begin{array}{l}\text { American Association } \\
\text { of Medical Physicists }\end{array}$ & http://aapm.org/pubs/reports/ \\
\hline AFSSAPS & $\begin{array}{l}\text { Agence Française de } \\
\text { Sécurité Sanitaire des } \\
\text { Produits de Santé }\end{array}$ & $\begin{array}{l}\text { http://www.afssaps.fr/Activites/Maintenance-et-controle- } \\
\text { qualite-des-DM/Referentiels-publies }\end{array}$ \\
\hline $\begin{array}{l}\text { AIEA / } \\
\text { IAEA }\end{array}$ & $\begin{array}{l}\text { Agence Internationale } \\
\text { de l'Énergie Atomique }\end{array}$ & $\begin{array}{l}\text { http://www-naweb.iaea.org/nahu/dmrp/publication.asp } \\
\text { http://www.iaea.org/Publications } \\
\text { https://rpop.iaea.org/RPOP/RPoP/Content/ } \\
\text { AdditionalResources/Publications/index.htm }\end{array}$ \\
\hline $\begin{array}{l}\text { ANAP / } \\
\text { MEAH }\end{array}$ & $\begin{array}{l}\text { Appui Santé et } \\
\text { Médico-Social }\end{array}$ & $\begin{array}{l}\text { http://www.anap.fr/nc/publications-outils/ } \\
\text { (mots clés = radiothérapie ou imagerie) }\end{array}$ \\
\hline ASN & $\begin{array}{l}\text { Autorité de Sûreté } \\
\text { Nucléaire }\end{array}$ & $\begin{array}{l}\text { http://www.asn.fr/index.php/S-informer/Publications/ } \\
\text { Guides-pour-les-professionnels/Activites-medicales } \\
\text { http://www.asn.fr/index.php/S-informer/Publications/La- } \\
\text { revue-Controle }\end{array}$ \\
\hline EFOMP & $\begin{array}{l}\text { European Federation } \\
\text { of Organisations for } \\
\text { Medical Physics }\end{array}$ & $\begin{array}{l}\text { http://www.efomp.org/professional-issues/policy- } \\
\text { statements.html }\end{array}$ \\
\hline ESTRO & $\begin{array}{l}\text { European Society for } \\
\text { Radiotherapy and } \\
\text { Oncology }\end{array}$ & $\begin{array}{l}\text { http://www.estro-education.org/publications/Pages/ } \\
\text { ESTROPhysicsBooklets.aspx } \\
\text { http://www.estro.org/estroactivities/Pages/Guidelines.aspx }\end{array}$ \\
\hline HAS & $\begin{array}{l}\text { Haute Autorité de } \\
\text { Santé }\end{array}$ & $\begin{array}{l}\text { http://www.has-sante.fr/portail/jcms/c_39085/ } \\
\text { recherche?portal=c_6737\&rechSpec=true\&id=c_39085\&t } \\
\text { ypesref=generated.GuideMethodologique\&typesref=FileD } \\
\text { ocument\&refine=true\&sort=pdate\&portlet=c_39085 }\end{array}$ \\
\hline INCA & $\begin{array}{l}\text { Institut National du } \\
\text { Cancer }\end{array}$ & http://www.e-cancer.fr/soins/radiotherapie \\
\hline IOMP & $\begin{array}{l}\text { International } \\
\text { Organization for } \\
\text { Medical Physics }\end{array}$ & $\begin{array}{l}\text { http://www.iomp.org/?q=node/5 (policy statements) } \\
\text { http://www.iomp.org/?q=node/34 (références } \\
\text { bibliographiques) }\end{array}$ \\
\hline IPEM & $\begin{array}{l}\text { Institute of Physics } \\
\text { and Engineering in } \\
\text { Medecine }\end{array}$ & $\begin{array}{l}\text { http://www.ipem.ac.uk/publications/ } \\
\text { freeipempublications/Pages/ForProfessionals.aspx }\end{array}$ \\
\hline IRSN & $\begin{array}{l}\text { Institut de } \\
\text { Radioprotection } \\
\text { et de Sûreté Nucléaire }\end{array}$ & $\begin{array}{l}\text { http://www.irsn.fr/FR/expertise/rapports_expertise/radio- } \\
\text { protection-homme/Pages/radioprotection_homme.aspx }\end{array}$ \\
\hline SFPM & $\begin{array}{l}\text { Société Française de } \\
\text { Physique Médicale }\end{array}$ & $\begin{array}{l}\text { http://www.sfpm.asso.fr/download/ } \\
\text { index.php?act=category\&id=3 }\end{array}$ \\
\hline
\end{tabular}

